\title{
Substantial risk of recurrence even after 5 recurrence-free years in early-stage hepatocellular carcinoma patients
}

Jihye Kim, Wonseok Kang, Dong Hyun Sinn, Geum-Youn Gwak, Yong-Han Paik, Moon Seok Choi, Joon Hyeok Lee, Kwang Cheol Koh, and Seung Woon Paik

Department of Medicine, Samsung Medical Center, Sungkyunkwan University School of Medicine, Seoul, Korea

\section{Graphical Abstract}

Risk of recurrence in early-stage hepatocellular carcinoma patients

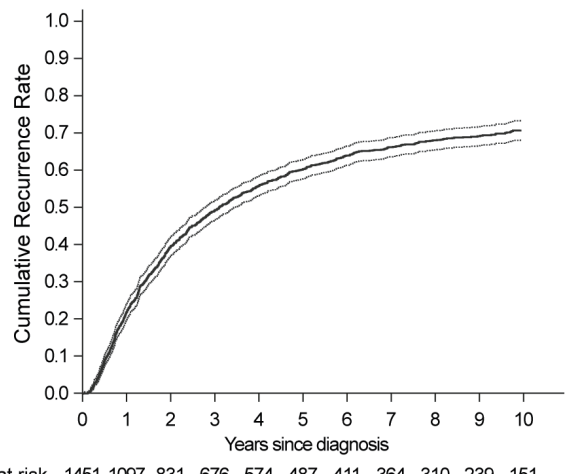

Number at risk $14511097831 \quad 676 \quad 574 \quad 487 \quad 411 \quad 364 \quad 310 \quad 239151$

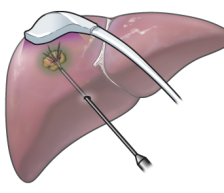

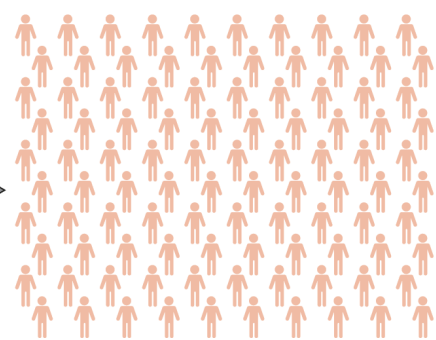

5 -year recurrence-free survivors following curative treatment for early-stage hepatocellular carcinoma

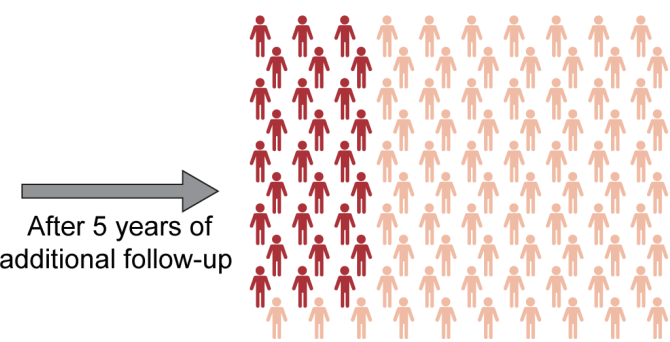

5 -year cumulative recurrence rate (5-10 years from initial diagnosis) was $27.0 \%$.

- HCC patients warrant continued secondary HCC surveillance, even after five recurrence-free years.

\section{Abbreviations:}

AFP, alpha-fetoprotein; ALBI, albumin-bilirubin; ALT, alanine aminotransferase; APRI, aspartate aminotransferase to platelet ratio index; AST, aspartate aminotransferase; AVT, antiviral therapy; BCLC, Barcelona Clinic Liver Cancer; $\mathrm{CT}$, computed tomography; FIB-4, fibrosis-4; HBV, hepatitis B virus; $\mathrm{HCC}$, hepatocellular carcinoma; HCV, hepatitis C virus; MELD, model for end-stage liver disease; MRI, magnetic resonance imaging; PIVKA-II, protein induced by vitamin Kabsence or antagonist-II; RFA, radiofrequency ablation

\section{Corresponding author : Dong Hyun Sinn}

Department of Medicine, Samsung Medical Center, Sungkyunkwan University School of Medicine, 81 Irwon-ro, Gangnam-gu, Seoul 06351, Korea

Tel: +82-2-3410-3409, Fax: +82-2-3410-6983

E-mail:dh.sinn@samsung.com

https://orcid.org/0000-0002-7126-5554 
Background/Aims: Although hepatocellular carcinoma (HCC) is notorious for its high recurrence rate, some patients do not experience recurrence for more than 5 years after resection or radiofrequency ablation for early-stage HCC. For those with five recurrence-free period, the risk of HCC recurrence within the next 5 years remains unknown.

Methods: A total of 1,451 consecutive patients (median, 55 years old; males, 79.0\%; hepatitis B virus-related, 79.3\%) with good liver function (Child-Pugh class A) diagnosed with early-stage HCC by Barcelona Clinic Liver Cancer Staging and received radiofrequency ablation or resection as an initial treatment between 2005 and 2010 were analyzed.

Results: During a median follow-up period of 8.1 years, 961 patients (66.2\%) experienced HCC recurrence. The cumulative recurrence rates increased to $39.7 \%, 60.3 \%$, and $71.0 \%$ at 2,5 , and 10 years, respectively, and did not reach a plateau. Five years after HCC diagnosis, 487 patients were alive without experiencing a recurrence. Among them, during a median of 3.9 additional years of follow-up (range, $0.1-9.0$ years), 127 patients (26.1\%) experienced recurrence. The next 5-year cumulative recurrence rate (5-10 years from initial diagnosis) was $27.0 \%$. Male sex, higher fibrosis-4 scores, and alpha-fetoprotein levels at 5 years were associated with later HCC recurrence among patients who did not experience recurrence for more than 5 years.

Conclusions: The HCC recurrence rate following 5 recurrence-free years after $\mathrm{HCC}$ treatment was high, indicating that HCC patients warrant continued HCC surveillance, even after 5 recurrence-free years. (Clin Mol Hepatol 2020;26:516528)

Keywords: Carcinoma, Hepatocellular; Treatment; Long-term; Recurrence

\section{Study Highlights}

- The recurrence rate of HCC in early-stage patients who received curative treatment was high, even after 5 recurrence-free years.

- Male sex, high fibrosis-4 scores, and alpha-fetoprotein levels were associated with later HCC recurrence, but even those without the above risk factors showed substantial recurrence rates.

- HCC patients warrant continued secondary HCC surveillance, even after 5 recurrence-free years.

\section{INTRODUCTION}

Hepatocellular carcinoma (HCC) generally develops in the setting of underlying chronic liver disease and carries high clinical and economic global burdens. ${ }^{1,2}$ HCC is notorious for its high recurrence rate, experienced by approximately two-thirds of patients within 5 years of curative treatment with resection or radiofrequency ablation (RFA). ${ }^{3-8}$ Nonetheless, some patients do not experience recurrence for more than 5 years after resection or RFA for early-stage HCC. In certain types of cancer (e.g., stomach or colon), 5 recurrence-free years suggests that the risk of recurrence is minimal. Hence, clinicians may stop routine secondary surveillance for tumor recurrence. ${ }^{9-13}$ However, patients with HCC usually have an underlying chronic disease, which may progress over time and de novo HCC may develop even after a long recurrence-free period. $^{14}$ This indicates that continued secondary surveillance for HCC may be needed even for those with long recurrence-free periods.
As cancer surveillance is costly and is not free from the risk of complications, false positivity, and/or radiation hazard, ${ }^{15}$ whether continued secondary surveillance for HCC after a long tumor recurrence-free period is required warrants further evaluation. With advances in HCC treatment and the management of underlying chronic liver disease, the number of long-term cancer survivors is also increasing ${ }^{16}$ giving rise to unmet needs in clinical practice. To the best of our knowledge, limited information is available regarding the risk and risk factors for HCC recurrence in patients who do not experience HCC recurrence for long periods (e.g., 5 years) after curative treatment with resection or RFA for early-stage HCC. This study aimed to identify the risk and risk factors for recurrence in early-stage HCC patients initially treated with resection or RFA, with special attention to those who did not experience recurrence for 5 years. 


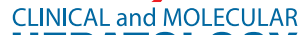

\section{HEPATOLOGY}

Volume_26 Number_4 October 2020

\section{PATIENTS AND METHODS}

\section{Study design, setting, and participants}

This study was a single-center, retrospective cohort study performed at Samsung Medical Center, Seoul, South Korea, using an HCC registry. The HCC registry is an electronic registry that records baseline clinical characteristics, tumor variables, and the initial treatment modalities of every newly-diagnosed HCC patient aged 18 years or older who received care at the Samsung Medical Center, Seoul, South Korea, in a prospective manner. A diagnosis of HCC was established either histologically or clinically, according to the regional HCC guidelines. ${ }^{17,18}$ We screened a total of 4,151 patients in the $\mathrm{HCC}$ registry and included patients with 1) early or very early-stage HCC (Barcelona Clinic Liver Cancer [BCLC] stage 0 or A), 2) who received resection and/or RFA as an initial treatment, and 3) had preserved liver function represented as ChildPugh class A. Finally, 1,451 consecutive, treatment-naïve, BCLC stage 0 or A HCC patients who were initially treated with resection and/or RFA were analyzed (Fig. 1). The study protocol was re- viewed and approved by the Institutional Review Board at Samsung Medical Center (IRB No. 2019-05-101). As the study used only de-identified data routinely collected during hospital visits, the requirement to obtain informed patient consent was waived.

\section{Variables, data sources, and measurements}

The primary outcome variable was tumor recurrence during follow-up. Tumor recurrence was diagnosed histologically or clinically using dynamic computed tomography (CT) and/or magnetic resonance imaging (MRI). After initial treatment with RFA or resection, the patients were usually monitored at 3 to 6 -month intervals using dynamic liver $\mathrm{CT}$ or MRI and/or tumor markers at the discretion of the physician in charge of the patient.

Data for the following baseline variables were collected from the Samsung Medical Center HCC registry recorded by trained abstractors: age at diagnosis, sex, comorbidities such as diabetes and receiving dialysis, etiology of the liver disease, Child-Pugh score, albumin-bilirubin (ALBI) grade, alanine aminotransferase (ALT) levels, aspartate aminotransferase (AST) levels, platelet

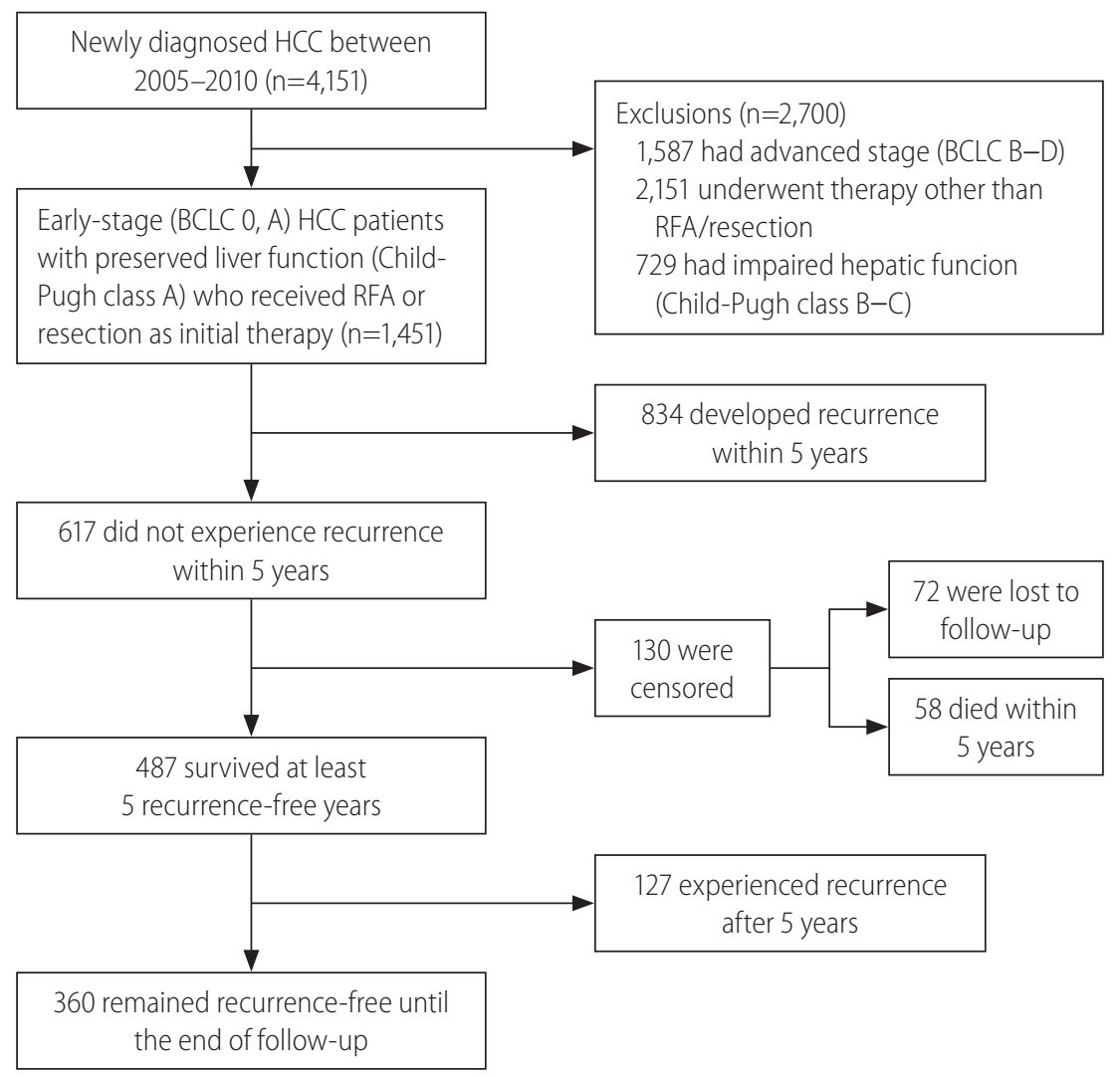

Figure 1. Study flow chart. HCC, hepatocellular carcinoma; BCLC, Barcelona Clinic Liver Cancer; RFA, radiofrequency ablation. 
Table 1. Baseline characteristics of the study population

\begin{tabular}{|c|c|c|c|c|}
\hline Variable & Overall $(n=1,451)$ & $\begin{array}{l}\text { Patients without recurrence } \\
\text { up to } 5 \text { years }(n=617)\end{array}$ & $\begin{array}{l}\text { Patients with recurrence } \\
\text { within } 5 \text { years }(n=834)\end{array}$ & $P$-value \\
\hline Age (years) & $55(49-63)$ & $54(48-62)$ & $56(50-63)$ & $0.010^{\S}$ \\
\hline$<60$ & $945(65.1)$ & $420(68.1)$ & $525(62.9)$ & $0.045^{\|}$ \\
\hline$\geq 60$ & $506(34.9)$ & 197 (31.9) & 309 (37.1) & \\
\hline Male & $1,147(79.0)$ & $472(76.5)$ & $675(80.9)$ & 0.040 \\
\hline Diabetes & $282(19.4)$ & $113(18.3)$ & $169(20.3)$ & 0.383 \\
\hline Etiology & & & & 0.326 \\
\hline $\mathrm{HBV}^{*}$ & $1,150(79.3)$ & $497(80.6)$ & $653(78.3)$ & \\
\hline Others $^{\dagger}$ & $301(20.7)$ & $120(19.4)$ & $181(21.7)$ & \\
\hline Liver cirrhosis & $692(47.7)$ & $243(39.4)$ & $449(53.8)$ & $<0.001$ \\
\hline Child-Pugh score & & & & 0.008 \\
\hline 5 & $1,132(78.0)$ & $502(81.4)$ & $630(75.5)$ & \\
\hline 6 & $319(22.0)$ & 115 (18.6) & $204(24.5)$ & \\
\hline ALBI grade & & & & $<0.001$ \\
\hline 1 & $838(57.8)$ & $392(63.5)$ & $446(53.5)$ & \\
\hline 2 & $613(42.2)$ & $225(36.5)$ & $388(46.5)$ & \\
\hline FIB-4 score & & & & $<0.001$ \\
\hline$<1.45$ & $248(17.1)$ & $137(22.2)$ & $111(13.3)$ & \\
\hline$\geq 1.45$ and $<3.25$ & $632(43.6)$ & $286(46.4)$ & $346(41.5)$ & \\
\hline$\geq 3.25$ & $571(39.4)$ & $194(31.4)$ & $377(45.2)$ & \\
\hline APRI & & & & $<0.001$ \\
\hline$<0.7$ & $643(44.3)$ & $324(52.5)$ & $319(38.2)$ & \\
\hline$\geq 0.7$ & $808(55.7)$ & $293(47.5)$ & $515(61.8)$ & \\
\hline MELD & & & & 0.023 \\
\hline$\leq 9$ & $1,141(78.6)$ & $503(81.5)$ & $638(76.5)$ & \\
\hline$\geq 10$ & $310(21.4)$ & $114(18.5)$ & $196(23.5)$ & \\
\hline $\operatorname{AFP}(\mathrm{ng} / \mathrm{mL})$ & $18.9(6.0-140.0)$ & $13.0(4.6-161.3)$ & $23.9(7.0-134.0)$ & 0.003 \\
\hline$<10$ & $574(39.6)$ & $279(45.2)$ & $295(35.4)$ & $<0.001$ \\
\hline$\geq 10$ & $877(60.4)$ & $338(54.8)$ & $539(64.6)$ & \\
\hline PIVKA-II $(m A U / m L)$ & & & & 0.354 \\
\hline$<40$ & $924(66.8)$ & $394(68.3)$ & $530(65.8)$ & \\
\hline$\geq 40$ & $459(33.2)$ & $183(31.7)$ & $276(34.2)$ & \\
\hline Tumor number & & & & 0.029 \\
\hline Single & $1,330(91.7)$ & $577(93.5)$ & $753(90.3)$ & \\
\hline Two & $107(7.4)$ & $38(6.2)$ & $69(8.3)$ & \\
\hline Three & $14(1.0)$ & $2(0.3)$ & $12(1.4)$ & \\
\hline Maximal tumor size (cm) & & & & 0.985 \\
\hline$\leq 2$ & $627(43.2)$ & $265(42.9)$ & $362(43.4)$ & \\
\hline$>2$ and $\leq 5$ & $695(47.9)$ & $297(48.1)$ & $398(47.7)$ & \\
\hline$>5$ & $129(8.9)$ & $55(8.9)$ & $74(8.9)$ & \\
\hline
\end{tabular}


Table 1. Continued

\begin{tabular}{|c|c|c|c|c|}
\hline Variable & Overall $(n=1,451)$ & $\begin{array}{l}\text { Patients without recurrence } \\
\text { up to } 5 \text { years }(n=617)\end{array}$ & $\begin{array}{l}\text { Patients with recurrence } \\
\text { within } 5 \text { years }(n=834)\end{array}$ & $P$-value \\
\hline BCLC stage & & & & 0.246 \\
\hline 0 & $442(30.5)$ & $198(32.1)$ & $244(29.3)$ & \\
\hline A & $1,009(69.5)$ & 419 (67.9) & $590(70.7)$ & \\
\hline Initial treatment & & & & $<0.001$ \\
\hline RFA & $649(44.7)$ & 199 (32.3) & $450(54.0)$ & \\
\hline Resection & $789(54.4)$ & $412(66.8)$ & $377(45.2)$ & \\
\hline RFA and resection & $13(0.9)$ & $6(1.0)$ & $7(0.8)$ & \\
\hline
\end{tabular}

Values are presented as median (quartile) or number (\%).

HBV, hepatitis B virus; ALBI, albumin-bilirubin; FIB-4 score, fibrosis-4 score; APRI, aspartate aminotransferase to platelet ratio; MELD, model for end-stage liver disease; AFP, alpha-fetoprotein; PIVKA-II, protein induced by vitamin K absence or antagonist-II; BCLC, Barcelona Clinic Liver Cancer; RFA, radiofrequency ablation.

*Eight cases had both HBV and hepatitis C virus infection.

${ }^{\dagger}$ Includes hepatitis $C$, alcohol, and non-B non-C.

"PIVKA-II results were missing in 68 patients.

${ }^{\S}$ Derived by the Mann-Whitney test using age as a continuous variable.

"Derived by the chi-squared test using age as a categorical variable.

counts, serum creatinine levels, alpha-fetoprotein (AFP) levels, protein induced by vitamin $\mathrm{K}$ absence or antagonist-II levels (PIVKA-II), tumor number, maximal tumor size, BCLC stage, and the initial treatment modality. We additionally collected the presence of liver cirrhosis at baseline and serum AST, ALT, platelet, and AFP levels at 5 years for patients who did not experience tumor recurrence for the first 5 years, using the electronic medical records of each patient at the Samsung Medical Center. Information on the use of antiviral agents before HCC diagnosis and during follow-up was collected. Liver cirrhosis was defined based on a combination of histology, imaging studies, and clinical features. The ALBI grades, fibrosis-4 (FIB-4) scores, AST to platelet ratio index (APRI), and the model for end-stage liver disease (MELD) scores were calculated using the original formulas (for the first three variables) or an updated formula (for MELD). ${ }^{19-22}$ The index visit was defined as the visit when the initial HCC diagnosis was made. The follow-up period was defined as the time from the index visit to tumor recurrence or the last follow-up, whichever came first (reference date: March 31, 2019). Death without tumor recurrence was censored at the time of the last follow-up.

\section{Statistical analyses}

The data are expressed as the median (interquartile range) for continuous variables and as the number (\%) of patients for categorical variables. The chi-squared test, Fisher's exact test, and
Mann-Whitney test were used to compare variables between the two groups. The factors associated with recurrence were tested using Cox regression analyses. Multivariable Cox regression analysis was performed to identify independent factors associated with tumor recurrence using variables with $P$-values less than 0.1 in univariable analysis. The analysis was performed for all included patients and for patients who did not have tumor recurrence for 5 years after HCC diagnosis. The cumulative HCC incidence was estimated using Kaplan-Meier methods and differences in the groups were compared using log-rank tests. All analyses involved two-sided tests of significance with $P$-values less than 0.05 considered statistically significant.

\section{RESULTS}

\section{Incidence and risk factors related to recurrence within 5 years}

The baseline characteristics of the study population and comparison between patients with and without recurrence for 5 years are shown in Table 1. The initial treatment included RFA in 649 patients $(44.7 \%)$, resection in 789 patients $(54.4 \%)$, and resection with intraoperative RFA in 13 patients $(0.9 \%)$. During a median follow-up period of 8.1 years, 961 patients (66.2\%) experienced HCC recurrence. Recurrence within the first 5 years of 


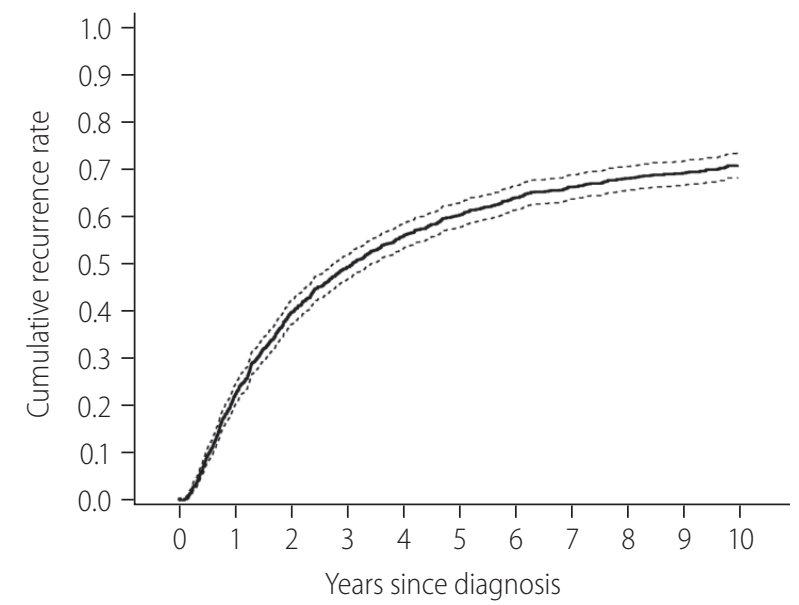

Number at risk 1,451 1,097 $831 \quad 676 \quad 574 \quad 487 \quad 411 \quad 364 \quad 310 \quad 239 \quad 151$

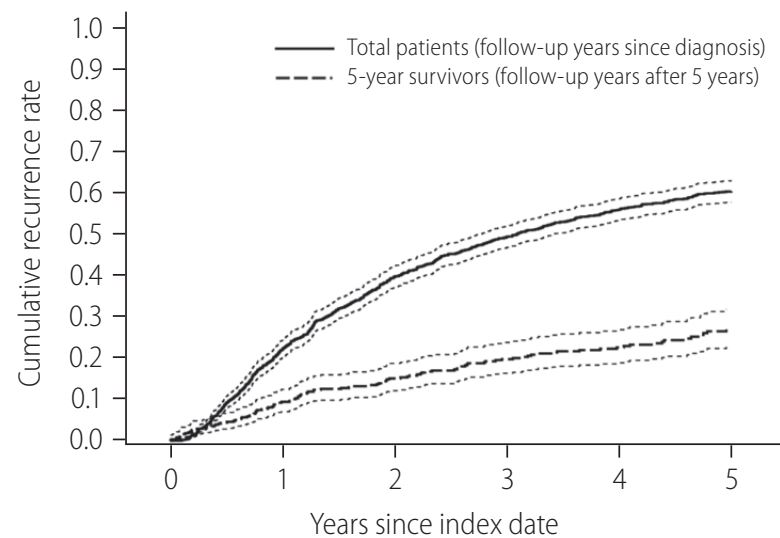

Number at risk

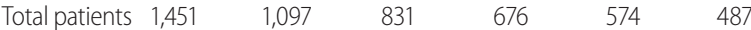

$\begin{array}{lllll}5 \text {-year survivors } 487 & 411 & 364 & 310 & 239\end{array}$

Figure 2. Cumulative recurrence rate in early-stage hepatocellular carcinoma patients treated with resection or radiofrequency ablation (dotted lines, $95 \%$ confidence intervals). (A) Whole study population. (B) Comparison of the next 5-year cumulative recurrence rate (5-10 years) among patients with 5 recurrence-free years (the dashed line) and the first 5 -year cumulative recurrence rate in the total patients (the solid line). The index date was the time of diagnosis for a total of 1,451 patients (indicated by the solid line), and 5 years after diagnosis the 487 survivors at 5-year follow-up without recurrence (indicated by the dashed line).

Table 2. Risk factors for recurrence within 5 years (overall patients, $n=1,451$ )

\begin{tabular}{|c|c|c|c|c|}
\hline \multirow{2}{*}{ Variable } & \multicolumn{2}{|c|}{ Unadjusted } & \multicolumn{2}{|c|}{ Multivariable } \\
\hline & HR (95\% Cl) & $P$-value & $\mathrm{HR}(95 \% \mathrm{Cl})$ & $P$-value \\
\hline Age $\geq 60$ vs. $<60$ (years) & $1.18(1.02-1.35)$ & 0.025 & $1.14(0.97-1.34)$ & 0.108 \\
\hline Male vs. female & $1.21(1.02-1.44)$ & 0.032 & $1.41(1.18-1.68)$ & $<0.001$ \\
\hline Diabetes & $1.01(0.85-1.20)$ & 0.893 & & \\
\hline HBV vs. others & $0.87(0.74-1.02)$ & 0.089 & $0.98(0.81-1.17)$ & 0.795 \\
\hline ALBI grade 2 vs. 1 & $1.32(1.16-1.52)$ & $<0.001$ & $1.23(1.06-1.42)$ & 0.007 \\
\hline FIB- $4 \geq 3.25$ vs. $<3.25$ & $1.46(1.28-1.68)$ & $<0.001$ & $1.14(0.97-1.33)$ & 0.116 \\
\hline AFP $\geq 10$ vs. $<10(\mathrm{ng} / \mathrm{mL})$ & $1.39(1.21-1.60)$ & $<0.001$ & $1.49(1.28-1.72)$ & $<0.001$ \\
\hline \multicolumn{5}{|l|}{ Tumor number } \\
\hline Single & Reference & & Reference & \\
\hline Two & $1.44(1.12-1.84)$ & 0.004 & $1.30(1.01-1.66)$ & 0.041 \\
\hline Three & $2.56(1.45-4.54)$ & 0.001 & $2.17(1.22-3.85)$ & 0.008 \\
\hline
\end{tabular}

Maximal tumor diameter $(\mathrm{cm})$

$\leq 2$

$>2$ and $\leq 5$

$>5$

Initial treatment modality

Resection

RFA*
Reference

$\begin{array}{ll}1.01(0.88-1.17) & 0.877 \\ 1.13(0.88-1.45) & 0.336\end{array}$

Reference

Reference

$1.78(1.55-2.04)$

$<0.001$

$1.68(1.45-1.94)$

$<0.001$

HR, hazard ratio; Cl, confidence interval; HBV, hepatitis B virus; ALBI, albumin-bilirubin; FIB-4 score, fibrosis-4 score; AFP, alpha-fetoprotein; RFA, radiofrequency ablation.

*Thirteen patients who received resection and radiofrequency ablation simultaneously were included in this category. 


\section{CLMEALAn MOLECULAR
HEPATOLOGY}

Volume_26 Number_4 October 2020

follow-up was observed in $57.5 \%(834 / 1,451)$ of the patients. The cumulative recurrence rates at 2, 5, and 10 years were $39.7 \%$, $60.3 \%$, and $71.0 \%$, respectively (Fig. $2 \mathrm{~A}$ ), without reaching a pla- teau. Patients experiencing recurrence within the first 5 years were older, more frequently male, and showed a higher proportion of liver cirrhosis, higher Child-Pugh scores, higher ALBI

Table 3. Comparison between patients with or without further recurrence after 5 recurrence-free years $(n=487)$

\begin{tabular}{|c|c|c|c|}
\hline Variable & $\begin{array}{l}\text { No recurrence until end of } \\
\text { follow-up }(n=360)\end{array}$ & $\begin{array}{l}\text { Recurrence after } 5 \text { years } \\
\qquad(n=127)\end{array}$ & $P$-value \\
\hline \multicolumn{4}{|l|}{ At baseline } \\
\hline Male & $268(74.4)$ & $106(83.5)$ & 0.038 \\
\hline Diabetes & $60(16.7)$ & $23(18.1)$ & 0.784 \\
\hline Etiology & & & 0.303 \\
\hline $\mathrm{HBV}^{*}$ & $312(86.7)$ & $105(82.7)$ & \\
\hline Others $^{\dagger}$ & $48(13.3)$ & $22(17.3)$ & \\
\hline Tumor number & & & 0.874 \\
\hline Single & $338(93.9)$ & $119(93.7)$ & \\
\hline Two & $21(5.8)$ & $8(6.3)$ & \\
\hline Three & $1(0.3)$ & $0(0.0)$ & \\
\hline Maximal tumor size $(\mathrm{cm})$ & & & 0.758 \\
\hline$\leq 2$ & $156(43.3)$ & $57(44.9)$ & \\
\hline$>2$ and $\leq 5$ & $174(48.3)$ & $62(48.8)$ & \\
\hline$>5$ & $30(8.3)$ & $8(6.3)$ & \\
\hline Initial treatment & & & 0.001 \\
\hline RFA & $94(26.1)$ & $44(34.6)$ & \\
\hline Resection & $265(73.6)$ & 78 (61.4) & \\
\hline RFA and resection & $1(0.3)$ & $5(3.9)$ & \\
\hline \multicolumn{4}{|l|}{ At 5 years since diagnosis } \\
\hline Age at 5-year & $58(53-65)$ & $59(54-66)$ & 0.270 \\
\hline$<60$ & $197(54.7)$ & $66(52.0)$ & 0.592 \\
\hline$\geq 60$ & $163(45.3)$ & $61(48.0)$ & \\
\hline 5-year ALBI grade & & & 0.054 \\
\hline 1 & $340(94.4)$ & $113(89.0)$ & \\
\hline 2 & $19(5.3)$ & $14(11.0)$ & \\
\hline 3 & $1(0.3)$ & $0(0.0)$ & \\
\hline 5-year FIB-4 score & & & $<0.001$ \\
\hline$<1.45$ & $73(20.3)$ & $16(12.6)$ & \\
\hline$\geq 1.45$ and $<3.25$ & $221(61.4)$ & $66(52.0)$ & \\
\hline$\geq 3.25$ & $66(18.3)$ & 45 (35.4) & \\
\hline 5-year AFP (ng/mL) & $2.3(1.7-3.5)$ & $3.4(2.6-5.8)$ & $<0.001$ \\
\hline$<10$ & $350(97.2)$ & $108(85.0)$ & $<0.001$ \\
\hline$\geq 10$ & $10(2.8)$ & $19(15.0)$ & \\
\hline
\end{tabular}

Values are presented as median (quartile) or number (\%).

$H B V$, hepatitis B virus; RFA, radiofrequency ablation; ALBI, albumin-bilirubin; FIB-4 score, fibrosis-4 score; AFP, alpha-fetoprotein.

*One case had both HBV and hepatitis C virus infection.

${ }^{\dagger}$ Includes hepatitis $C$, alcohol, and non-B non-C. 
grades, higher FIB-4 scores, higher APRI scores, higher MELD scores, and higher AFP levels. The proportion of multiple tumors and patients receiving RFA as initial treatment was also higher in patients with recurrence within 5 years than in patients who did not experience recurrence within 5 years (Table 1). In Cox regression analysis with multivariable adjustment, HCC recurrence within 5 years was significantly associated with male sex, higher ALBI grades, higher AFP levels ( $\geq 10 \mathrm{ng} / \mathrm{mL})$, multiple tumors, and treatment modality (Table 2). Even in the analysis with BCLC instead of tumor number and size, the results were consistent (Supplementary Table 1).

\section{Incidence and risk factors related to recurrence after 5 years}

There were 487 patients who were alive, had no recurrence, and were not lost to follow-up 5 years after HCC diagnosis. They received a median of 3.9 additional years of follow-up (range, 0.1-9.0 years). The cumulative recurrence rate between 5 and 10 years after HCC diagnosis was $27.0 \%$ (95\% confidence interval, $22.7-31.8 \%$ ). The surveillance interval and modality for patients with 5 recurrence-free years varied. The most frequent surveillance interval was 6 months (49.4\%) with CT as the surveillance modality $(77.1 \%)$ at the time of recurrence. The HCC status at the time of recurrence was BCLC 0 stage in $55.4 \%$ and $A$ stage in

Table 4. Risk factors for further recurrence among patients without recurrence within 5 years $(n=487)$

\begin{tabular}{|c|c|c|c|c|}
\hline \multirow{2}{*}{ Variable } & \multicolumn{2}{|c|}{ Unadjusted } & \multicolumn{2}{|c|}{ Multivariable } \\
\hline & HR $(95 \%$ Cl) & $P$-value & $\mathrm{HR}(95 \% \mathrm{Cl})$ & $P$-value \\
\hline Age at 5-year $\geq 60$ vs. $<60$ (years) & $1.27(0.89-1.80)$ & 0.187 & & \\
\hline Male vs. female & $1.70(1.06-2.71)$ & 0.027 & $2.01(1.24-3.24)$ & 0.004 \\
\hline Diabetes & $1.06(0.68-1.67)$ & 0.794 & & \\
\hline HBV vs. others & $0.70(0.44-1.10)$ & 0.124 & & \\
\hline \multicolumn{5}{|l|}{ Tumor number } \\
\hline Single & Reference & & & \\
\hline Two or three & $1.38(0.67-2.83)$ & 0.377 & & \\
\hline \multicolumn{5}{|l|}{ Maximal tumor diameter (cm) } \\
\hline$\leq 2(n=213)$ & Reference & & & \\
\hline$>2$ and $\leq 5(n=236)$ & $1.07(0.75-1.53)$ & 0.714 & & \\
\hline$>5(n=38)$ & $0.67(0.32-1.41)$ & 0.290 & & \\
\hline \multicolumn{5}{|l|}{ Initial treatment modality } \\
\hline Resection $(n=343)$ & Reference & & Reference & \\
\hline $\operatorname{RFA}^{*}(n=144)$ & $1.59(1.11-2.27)$ & 0.011 & $1.32(0.91-1.92)$ & 0.142 \\
\hline \multicolumn{5}{|l|}{ 5-year ALBI grade } \\
\hline $1(n=453)$ & Reference & & Reference & \\
\hline$\geq 2(n=34)$ & $2.08(1.19-3.64)$ & 0.010 & $0.99(0.51-1.92)$ & 0.981 \\
\hline \multicolumn{5}{|l|}{ 5-year FIB-4 } \\
\hline$<3.25(n=376)$ & Reference & & Reference & \\
\hline$\geq 3.25(n=111)$ & $1.97(1.37-2.84)$ & $<0.001$ & $1.69(1.12-2.54)$ & 0.012 \\
\hline \multicolumn{5}{|l|}{ 5-year AFP (ng/mL) } \\
\hline$<10(n=458)$ & Reference & & Reference & \\
\hline$\geq 10(n=29)$ & $4.28(2.62-6.98)$ & $<0.001$ & $3.37(1.92-5.93)$ & $<0.001$ \\
\hline
\end{tabular}

HR, hazard ratio; Cl, confidence interval; HBV, hepatitis B virus; RFA, radiofrequency ablation; ALBI, albumin-bilirubin; FIB-4 score, fibrosis-4 score; AFP, alphafetoprotein.

*This category includes patients who received resection and radiofrequency ablation simultaneously. 
$37.3 \%$ of the patients. Patients whose disease did not recur until the last follow-up differed by sex, initial treatment modality, FIB-4 scores, and AFP levels at five years compared to patients who experienced recurrence (Table 3). In multivariable regression analysis, HCC recurrence among those without recurrence for the first 5 years was associated with male sex, FIB-4 scores above 3.25 , and elevated AFP levels above $10 \mathrm{ng} / \mathrm{mL}$ at 5 -year follow-up (Table 4).

The cumulative HCC recurrence rate at each year was higher within the first 5 years than within the 5-10 years period after HCC diagnosis (Fig. 2B). When the 487 patients who survived beyond 5 years without recurrence were classified according to a number of identified risk factors (male sex, FIB-4 scores above 3.25, and AFP levels above $10 \mathrm{ng} / \mathrm{mL}$ at 5 -year), the cumulative $\mathrm{HCC}$ recurrence rates over the next 5 years $(5-10$ years after $\mathrm{HCC}$ diagnosis) were $71.4 \%, 45.0 \%, 24.5 \%$, and $10.3 \%$ in patients with three risk factors, two risk factors, one risk factor, and no risk factors, respectively $(P<0.001$, Fig. 3).

Among the studied population, 100 patients (6.9\%) did not have hepatitis B virus (HBV), hepatitis C virus (HCV) or cirrhosis of any etiology. Forty-two patients experienced HCC recurrence within the first 5 years, and 37 patients survived 5 years or more without recurrence. Among 37 patients with a recurrence-free survival of 5 years or more, six patients experienced recurrence 5.7 to 8.0 years after the initial diagnosis with a 5 -year cumulative incidence rate of $20.0 \%$.

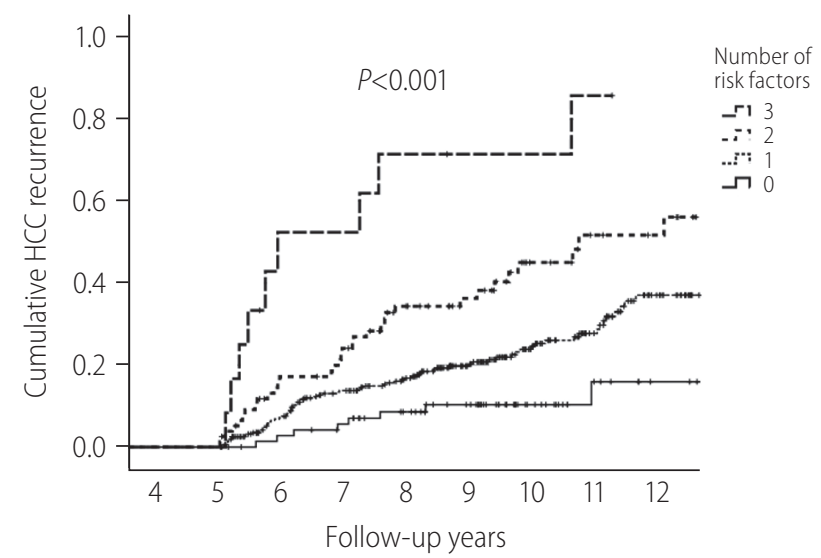

Number of risk

$\begin{array}{ccccccccc}3 & 12 & 5 & 5 & 3 & 3 & 1 & 0 & 0 \\ 2 & 78 & 61 & 55 & 40 & 33 & 19 & 13 & 11 \\ 1 & 322 & 275 & 238 & 210 & 158 & 105 & 72 & 37 \\ 0 & 75 & 70 & 66 & 57 & 46 & 25 & 14 & 9\end{array}$

Figure 3. HCC recurrence after 5 recurrence-free years according to the number of verified risk factors $(n=487)$. HCC, hepatocellular carcinoma.

\section{DISCUSSION}

In this study, the 5-year recurrence rate was high (60.3\%) in early-stage (BCLC 0 or A) HCC patients initially treated with resection and/or RFA. Although the HCC recurrence rate gradually decreased with increasing follow-up time, the cumulative $\mathrm{HCC}$ recurrence did not reach a plateau in 10 years of follow-up (Fig. 2A). Among patients who did not experience HCC recurrence within the first 5 years, the next 5-year (5-10 years) cumulative recurrence rate was not low $(27.0 \%)$. The independent risk factors for recurrence within the first 5 years were male sex, higher ALBI grades, higher AFP levels, multiple tumors, and treatment with RFA. In patients with 5-year recurrence-free survival, the independent risk factors for future recurrence were male sex and higher FIB- 4 scores and AFP levels at 5 years. Among the patients without recurrence for 5 years, the risk of recurrence within the next 5 years (5-10 years) was very high for patients with three risk factors (male, high FIB-4 score, and high AFP level; 71.4\%), and was not low for patients without risk factors (female, low FIB-4 score, and low AFP level; 10.3\%).

The high recurrence rates found in this study differed from those of other common gastrointestinal cancers. The cumulative incidence rate of gastric cancer following curative resection (3.7\% at 10 years and $5.4 \%$ at 20 years) is similar to the incidence of primary gastric cancer (1.3\% per year worldwide). ${ }^{23,24}$ In a study of 1,058 patients receiving curative intent gastrectomy for T1-2N0 gastric adenocarcinoma in the United States and China, 7\% of the patients experienced recurrences during a median follow-up of over 5 years, almost all of which occurred between 6 months and 3 years postoperatively..$^{25}$ There is no available evidence supporting routine surveillance for asymptomatic cancer recurrence after curative gastrectomy. Hence, routine intensive radiological evaluation or endoscopy for secondary cancer surveillance is not recommended, especially after 5 recurrence-free years. ${ }^{12,26}$ Colon cancer patients resected with curative-intent showed low recurrence rates 5 to 10 years after initial surgery (2.9\% for local recurrence and $4.3 \%$ for distant metastasis), compared to the 1-year cumulative incidence of primary colon cancer worldwide $(2.3 \%) .{ }^{27,28}$ Therefore, long-term intensive secondary surveillance for colorectal cancer is not recommended and colonoscopies at 5-year intervals are recommended starting 4 years after surgery. $13,29,30$

However, in the case of HCC, the long-term recurrence rates reported after curative treatment were quite high, as in this study. In a Western study focusing on actual 10-year survivors after cu- 
rative-intent resection for HCC, 11 out of 50 patients (22\%) developed recurrence after the first 5 years while $62 \%$ experienced recurrence within 5 years. ${ }^{31}$ Among 1,294 Japanese patients treated with RFA for primary HCC, the 5- and 10-year distant recurrence rates were $74.8 \%$ and $80.8 \%$, respectively. ${ }^{32}$ Currently, in Korea, once a person receives a cancer diagnosis, he/she is registered to the National Cancer Registry with a C-code that provides additional insurance benefits to cancer patients for the first 5 years. Considering the high recurrence rate, $\mathrm{HCC}$ patients need continued surveillance even after a 5 -year recurrence-free period. This needs to be considered for HCC patients in terms of the national insurance policy in Korea.

Because the early detection of recurrence allows the possibility of the reapplying curative treatment modalities, post-treatment monitoring is recommended frequently enough to detect recurrence as early as possible. ${ }^{33}$ The risk of HCC was lower after five recurrence-free years, compared to the first 5-years, indicating that a different surveillance strategy might be needed for those with long-term recurrence-free periods. In this study, the selection of 5 recurrence-free years was arbitrary, considering the current insurance policy in Korea, and the time point was not selected based on recurrence risk. The exact time point (e.g., after 2 years, 4 years or 6 years after treatment) when different surveillance strategy is needed is not known. Additionally, the surveillance interval and the surveillance modality after five recurrence-free years are important issues as well. In this study, the surveillance intervals and methods were at the discretion of the physician in charge of the patient. Hence, further analysis of these factors could not be performed. Thus, the proper surveillance strategies for those with 5 recurrence-free years warrants further evaluation.

In previous studies assessing the risk factors for HCC recurrence, late recurrence (usually defined as tumor recurrence after 24 months) was related to underlying chronic liver disease, whereas early recurrence (usually defined as tumor recurrence within first 24 months) was associated with tumor-related factors (i.e., tumor size, tumor number, serum tumor marker, peripheral invasion, and vascular invasion) and treatment modality. ${ }^{34-40}$ Consistent with those findings, our study also found that tumor factors or treatment modality were no longer independent factors for future HCC recurrence in $\mathrm{HCC}$ patients with 5 recurrence-free years while they were independent risk factors for $\mathrm{HCC}$ recurrence in the first 5 years.

Although RFA and resection are considered curative-intent treatments for early-stage HCC, the baseline characteristics of the patients in our study differed significantly between those receiv- ing RFA and resection (Supplementary Table 2), and these two groups showed different risks of tumor recurrence (Supplementary Fig. 1). In the multivariable-adjusted analysis, the risk of recurrence was different for the first 5 years according to the treatment but did not differ after 5 years in this study. However, considering the study size (138 patients who received RFA and survived more than 5 years without recurrence), this finding needs further evaluation in a larger sample.

Notably, the FIB-4 score at 5 years, which was developed and validated as a noninvasive marker for predicting liver fibrosis and cirrhosis, ${ }^{41-43}$ was an independent factor for HCC recurrence. FIB-4 is considered a promising prognostic factor for monitoring HCC patient survival and recurrence after treatment. ${ }^{44}$ Although the patients had significantly different recurrence rates according to the presence of liver cirrhosis (Supplementary Fig. 2), we used FIB-4 to estimate the fibrosis burden in the overall cohort, as histological information was missing for patients who received RFA. When the analysis was limited to 802 patients who underwent resection and had histologic information, advanced fibrosis defined by histology was a risk factor for recurrence within 5 years (Supplementary Table 3). Advanced fibrosis was also a risk factor for later recurrence among 349 patients with 5 recurrence-free years (Supplementary Table 4).

Using these risk factors, we wanted to determine if there was any specific population with an extremely low risk for recurrence after 5 years, for whom secondary surveillance may not be necessary. However, there were no subgroups with extremely low risk of future recurrence among early-stage HCC patients treated with resection or RFA. Even in patients with 5 recurrence-free years, and without any identified risk factors for $\mathrm{HCC}$ recurrence, the risk of later HCC development was substantial (5-year cumulative recurrence rate of $10.3 \%$ for the next 5 years). Although direct comparison is difficult, this rate was lower than the annual incidence rate of $\mathrm{HCC}$ in cirrhotic patients $(3-7 \%)^{45-47}$ but was higher than the yearly incidence rate of HCC $(0.7-1.7 \%)$ in chronic hepatitis B patients who received antiviral therapy (AVT) for 5 years. ${ }^{48,49}$ For those with any risk factors, the HCC recurrence risk for the next 5 years (5-10 years) was substantial (24.5-71.4\%). We also noticed that the HCC recurrence risk was substantial among 100 patients without HBV, HCV, or cirrhosis, even after 5 recurrence-free years. Although the studied sample size was small, this finding indicates that continued HCC surveillance is warranted for long-term survivors of HCC even if they do not have HBV, HCV, or cirrhosis. Our findings indicate that secondary surveillance is necessary for all patients, including patients with 5 re- 
currence-free years, initially treated with resection or RFA for early-stage HCC.

There were several limitations to this study. Several factors that might be associated with future HCC recurrence were not analyzed in this study. HBV was the most common etiology (79.3\%) in the studied population, and AVT for HBV can modify the risk of HCC recurrence. ${ }^{50}$ In this study, 514 patients (44.7\%) were using or started AVT at HCC diagnosis. Among 636 patients who were not using AVT at HCC diagnosis, 190 started AVT during followup at different times before HCC recurrence. As the study cohort was not comprised of HBV patients only, and AVT was started at different times with different virological outcomes, the impact of AVT could not be assessed in this study. Smoking status, alcohol use, and AVT for HCV infection were also not analyzed. Some of these variables had high rates of missing data and the measurement methods used were not standardized during the follow-up periods. Hence, there is a possibility that unidentified factors could be associated with later HCC recurrence. This study was conducted in a single referral center in a high HBV endemic area, limiting the generalizability to other areas where the major etiology of HCC is different. In addition, although high recurrence rates indicate that secondary HCC surveillance may be cost-effective and may lead to mortality reduction compared to non-surveillance, the actual risk and benefit of secondary HCC surveillance have not been studied in controlled trials. Therefore, the optimal intervals and methods for secondary HCC surveillance also remain to be determined.

In summary, the HCC recurrence rates were high, even after 5 recurrence-free years following HCC treatment. Therefore, HCC patients warrant continued HCC surveillance, even after 5 recurrence-free years, especially in men with high FIB-4 scores and elevated AFP levels at 5-year follow-up. Studies to determine the optimal secondary surveillance methods for these long-term cancer survivors are needed.

\section{Authors' contribution}

Conception and design: Dong Hyun Sinn. Acquisition, analysis, or interpretation of data: Jihye Kim. Drafting and writing: Jihye Kim and Dong Hyun Sinn. Resources and critical revision of the manuscript for important intellectual content: Wonseok Kang, Dong-Hyun Sinn, Moon Seok Choi, Geum-Youn Gwak, Yong-Han Paik, Joon Hyeok Lee, Kwang Cheol Koh, and Seung Woon Paik. Study supervision: Dong-Hyun Sinn. All authors approved the final submission.

\section{Conflicts of Interest}

The authors have no conflicts to disclose.

\section{SUPPLEMENTAL MATERIAL}

Supplementary material is available at Clinical and Molecular Hepatology website (http://www.e-cmh.org).

\section{REFERENCES}

1. Forner A, Llovet JM, Bruix J. Hepatocellular carcinoma. Lancet 2012;379:1245-1255

2. Sayiner M, Golabi P, Younossi ZM. Disease burden of hepatocellular carcinoma: a global perspective. Dig Dis Sci 2019;64:910-917.

3. Roayaie S, Blume IN, Thung SN, Guido M, Fiel MI, Hiotis S, et al. A system of classifying microvascular invasion to predict outcome after resection in patients with hepatocellular carcinoma. Gastroenterology 2009;137:850-855.

4. Shen YC, Hsu C, Chen LT, Cheng CC, Hu FC, Cheng AL. Adjuvant interferon therapy after curative therapy for hepatocellular carcinoma (HCC): a meta-regression approach. J Hepatol 2010;52:889-894.

5. Belghiti J, Panis Y, Farges O, Benhamou JP, Fekete F. Intrahepatic recurrence after resection of hepatocellular carcinoma complicating cirrhosis. Ann Surg 1991;214:114-117.

6. Kumada T, Nakano S, Takeda I, Sugiyama K, Osada T, Kiriyama S, et al. Patterns of recurrence after initial treatment in patients with small hepatocellular carcinoma. Hepatology 1997;25:87-92.

7. Montorsi M, Santambrogio R, Bianchi P, Donadon M, Moroni E, Spinelli $A$, et al. Survival and recurrences after hepatic resection or radiofrequency for hepatocellular carcinoma in cirrhotic patients: a multivariate analysis. J Gastrointest Surg 2005;9:62-67; discussion 67-68.

8. Rhim H, Lim HK. Radiofrequency ablation of hepatocellular carcinoma: pros and cons. Gut Liver 2010;4 Suppl 1(Suppl 1):S113-S118.

9. Baiocchi GL, D'Ugo D, Coit D, Hardwick R, Kassab P, Nashimoto A, et al. Follow-up after gastrectomy for cancer: the Charter Scaligero Consensus Conference. Gastric Cancer 2016;19:15-20.

10. Aurello P, Petrucciani N, Antolino L, Giulitti D, D'Angelo F, Ramacciato $\mathrm{G}$. Follow-up after curative resection for gastric cancer: is it time to tailor it? World J Gastroenterol 2017;23:3379-3387.

11. Fahy BN. Follow-up after curative resection of colorectal cancer. Ann Surg Oncol 2014;21:738-746.

12. National Comprehensive Cancer Network (NCCN). Clinical Practice Guidelines in Oncology (NCCN Guidelines) - Gastric Cancer. Version 2.2019. Jun 3, 2019. NCCN web site, <https://www.nccn.org/ 
professionals/physician_gls/pdf/gastric.pdf>. Accessed 19 Jul 2019.

13. National Comprehensive Cancer Network (NCCN). Clinical Practice Guidelines in Oncology (NCCN Guidelines) - Colon Cancer. Version 2.2019. May 15, 2019. NCCN web site, <https://www.nccn.org/ professionals/physician_gls/pdf/colon.pdf>. Accessed 19 Jul 2019.

14. Tung-Ping Poon R, Fan ST, Wong J. Risk factors, prevention, and management of postoperative recurrence after resection of hepatocellular carcinoma. Ann Surg 2000;232:10-24.

15. Korean Liver Cancer Study Group (KLCSG); National Cancer Center, Korea (NCC). 2014 KLCSG-NCC Korea practice guideline for the management of hepatocellular carcinoma. Gut Liver 2015;9:267317.

16. Kim BH, Park JW. Epidemiology of liver cancer in South Korea. Clin Mol Hepatol 2018;24:1-9.

17. Park JW; Korean Liver Cancer Study Group and National Cancer Center. Practice guideline for diagnosis and treatment of hepatocellular carcinoma. Korean J Hepatol 2004;10:88-98.

18. Jang JY, Lee JS, Kim HJ, Shim JJ, Kim JH, Kim BH, et al. The general rules for the study of primary liver cancer. J Liver Cancer 2017;17:1944.

19. Johnson PJ, Berhane S, Kagebayashi C, Satomura S, Teng M, Reeves $\mathrm{HL}$, et al. Assessment of liver function in patients with hepatocelIular carcinoma: a new evidence-based approach-the ALBI grade. J Clin Oncol 2015;33:550-558.

20. Sterling RK, Lissen E, Clumeck N, Sola R, Correa MC, Montaner J, et al. Development of a simple noninvasive index to predict significant fibrosis in patients with HIV/HCV coinfection. Hepatology 2006;43:1317-1325.

21. Wai CT, Greenson JK, Fontana RJ, Kalbfleisch JD, Marrero JA, Conjeevaram HS, et al. A simple noninvasive index can predict both significant fibrosis and cirrhosis in patients with chronic hepatitis C. Hepatology 2003;38:518-526.

22. United Network for Organ Sharing (UNOS). Important policy notice: clerical changes for implementation of adding serum sodium to the MELD Score. UNOS web site, <https://optn.transplant.hrsa.gov/media/1575/policynotice_20151101.pdf>. Accessed 5 Mar 2020.

23. Hanyu T, Wakai A, Ishikawa T, Ichikawa H, Kameyama H, Wakai T. Carcinoma in the remnant stomach during long-term follow-up after distal gastrectomy for gastric cancer: analysis of cumulative incidence and associated risk factors. World J Surg 2018;42:782-787.

24. International Agency for Research on Cancer (IARC). Stomach. Source: Globocan 2018. IARC web site, <http://gco.iarc.fr/today/ data/factsheets/cancers/7-Stomach-fact-sheet.pdf>. Accessed 19 Jul 2019.

25. Cao L, Selby LV, Hu X, Zhang Y, Janjigian YY, Tang L, et al. Risk factors for recurrence in T1-2N0 gastric cancer in the United States and China. J Surg Oncol 2016;113:745-749.

26. Nilsson M. Postgastrectomy follow-up in the West: evidence base, guidelines, and daily practice. Gastric Cancer 2017;20(Suppl 1):135140.

27. Bouvier AM, Launoy G, Bouvier V, Rollot F, Manfredi S, Faivre J, et al. Incidence and patterns of late recurrences in colon cancer patients. Int J Cancer 2015;137:2133-2138.

28. International Agency for Research on Cancer (IARC). Colorectal cancer. Source: Globocan 2018. IARC web site, <http://gco.iarc. fr/today/data/factsheets/cancers/10_8_9-Colorectum-fact-sheet. pdf>. Accessed 19 Jul 2019.

29. Kahi CJ, Boland CR, Dominitz JA, Giardiello FM, Johnson DA, Kaltenbach $\mathrm{T}$, et al. Colonoscopy surveillance after colorectal cancer resection: recommendations of the US Multi-Society Task Force on colorectal cancer. Gastroenterology 2016;150:758-768.e11.

30. Rusiecki J, Cifu AS. Colonoscopy surveillance after colorectal cancer resection. JAMA 2017;318:2346-2347.

31. Zheng J, Kuk D, Gönen M, Balachandran VP, Kingham TP, Allen PJ, et al. Actual 10-year survivors after resection of hepatocellular carcinoma. Ann Surg Oncol 2017;24:1358-1366.

32. Shiina S, Tateishi R, Arano T, Uchino K, Enooku K, Nakagawa H, et al. Radiofrequency ablation for hepatocellular carcinoma: 10-year outcome and prognostic factors. Am J Gastroenterol 2012;107:569577; quiz 578.

33. Korean Liver Cancer Association; National Cancer Center. 2018 Korean Liver Cancer Association-National Cancer Center Korea practice guidelines for the management of hepatocellular carcinoma. Gut Liver 2019;13:227-299.

34. Cho JY, Choi MS, Lee GS, Sohn W, Ahn J, Sinn DH, et al. Clinical significance and predictive factors of early massive recurrence after radiofrequency ablation in patients with a single small hepatocelIular carcinoma. Clin Mol Hepatol 2016;22:477-486.

35. Hirokawa F, Hayashi M, Asakuma M, Shimizu T, Inoue Y, Uchiyama K. Risk factors and patterns of early recurrence after curative hepatectomy for hepatocellular carcinoma. Surg Oncol 2016;25:24-29.

36. Imamura H, Matsuyama Y, Tanaka E, Ohkubo T, Hasegawa K, Miyagawa $S$, et al. Risk factors contributing to early and late phase intrahepatic recurrence of hepatocellular carcinoma after hepatectomy. J Hepatol 2003;38:200-207.

37. Kim JM, Yi NJ, Kwon CHD, Lee KW, Suh KS, Joh JW. Early disseminated recurrence after liver resection in solitary hepatocellular carcinoma. Ann Surg Treat Res 2018;94:129-134.

38. Poon RT, Fan ST, Ng IO, Lo CM, Liu CL, Wong J. Different risk factors and prognosis for early and late intrahepatic recurrence after resection of hepatocellular carcinoma. Cancer 2000;89:500-507.

39. Xu XF, Xing H, Han J, Li ZL, Lau WY, Zhou YH, et al. Risk factors, patterns, and outcomes of late recurrence after liver resection for hepatocellular carcinoma: a multicenter study from China. JAMA Surg 2019;154:209-217.

40. Yamamoto $Y$, Ikoma $H$, Morimura R, Konishi H, Murayama $Y$, Kom- 


\section{CLINICAL and MOLECULAR
HEPATOLOGY}

atsu $\mathrm{S}$, et al. Optimal duration of the early and late recurrence of hepatocellular carcinoma after hepatectomy. World J Gastroenterol 2015;21:1207-1215.

41. Sterling RK, Lissen E, Clumeck N, Sola R, Correa MC, Montaner J, et al. Development of a simple noninvasive index to predict significant fibrosis in patients with HIV/HCV coinfection. Hepatology 2006;43:1317-1325.

42. Vallet-Pichard A, Mallet V, Nalpas B, Verkarre V, Nalpas A, DhalluinVenier $V$, et al. FIB-4: an inexpensive and accurate marker of fibrosis in HCV infection. comparison with liver biopsy and fibrotest. Hepatology 2007;46:32-36.

43. Kim BK, Kim DY, Park JY, Ahn SH, Chon CY, Kim JK, et al. Validation of FIB-4 and comparison with other simple noninvasive indices for predicting liver fibrosis and cirrhosis in hepatitis B virus-infected patients. Liver Int 2010;30:546-553.

44. Zhang Y, Wang R, Yang X. FIB-4 index serves as a noninvasive prognostic biomarker in patients with hepatocellular carcinoma: a metaanalysis. Medicine (Baltimore) 2018;97:e13696.

45. Santi V, Trevisani F, Gramenzi A, Grignaschi A, Mirici-Cappa F, Del Poggio $P$, et al. Semiannual surveillance is superior to annual surveil- lance for the detection of early hepatocellular carcinoma and patient survival. J Hepatol 2010;53:291-297.

46. Tzartzeva K, Obi J, Rich NE, Parikh ND, Marrero JA, Yopp A, et al. Surveillance imaging and alpha fetoprotein for early detection of hepatocellular carcinoma in patients with cirrhosis: a meta-analysis. Gastroenterology 2018;154:1706-1718.e1.

47. Fattovich G, Stroffolini T, Zagni I, Donato F. Hepatocellular carcinoma in cirrhosis: incidence and risk factors. Gastroenterology 2004;127(5 Suppl 1):S35-S50.

48. Papatheodoridis GV, Idilman R, Dalekos GN, Buti M, Chi H, van Boemmel $F$, et al. The risk of hepatocellular carcinoma decreases after the first 5 years of entecavir or tenofovir in Caucasians with chronic hepatitis B. Hepatology 2017;66:1444-1453.

49. Kim BG, Park NH, Lee SB, Jeon S, Park JH, Jung SW, et al. The risk of hepatocellular carcinoma within and beyond the first 5 years of entecavir in Korean patients with chronic hepatitis B. Liver Int 2018;38:2269-2276.

50. Kim JH, Sinn DH, Kim K, Kim H, Gwak GY, Paik YH, et al. Lamivudine versus entecavir for newly diagnosed hepatitis B virus-related hepatocellular carcinoma. Gut Liver 2016;10:939-947. 